\title{
Efficient Methods for Multi-agent Multi-issue Negotiation: Allocating Resources
}

\author{
Mengxiao $\mathrm{Wu}^{1}$, Mathijs de Weerdt ${ }^{2}$, and Han La Poutré ${ }^{1}$ \\ ${ }^{1}$ Centre for Mathematics and Computer Science (CWI), Amsterdam, The Netherlands \\ wu@cwi.nl, hlp@cwi.nl \\ 2 Delft University of Technology, Delft, The Netherlands \\ M.M.deWeerdtatudelft.nl
}

\begin{abstract}
In this paper, we present an automated multi-agent multi-issue negotiation solution to solve a resource allocation problem. We use a multilateral negotiation model, by which three agents bid sequentially in consecutive rounds till some deadline. Two issues are bundled and negotiated concurrently, so winwin opportunities can be generated as trade-offs exist between issues. We develop negotiation strategies of the agents under an incomplete information setting. The strategies are composed of a Pareto-optimal-search method and concession strategies. An important technical contribution of this paper lies in the development of the Pareto-optimal-search method for three-agent multilateral negotiation. Moreover, we present the identification of agreements and Pareto-optimal outcomes achieved by our methods in mathematical proof. We show through computer experiments that using the tractable heuristic of Pareto-optimal-search combined with well-designed concession strategies by agents results in (near) Pareto-optimal outcomes.
\end{abstract}

\section{Introduction}

With the rapid development of multi-agent systems, automated negotiation has been widely used to solve coordination and cooperation problems in complex systems. The potentially time-consuming negotiation process is delegated to autonomous software agents who conduct the negotiation on behalf of rational players. In this paper, we propose an automated multi-agent multi-issue negotiation solution to solve a resource allocation problem. In contrast to most previous work on two-player multi-issue negotiation (e.g., [1]) or multi-player single-issue negotiation (e.g., [2]), the negotiation model presented in this work is a multi-player multi-issue negotiation model in particular three-player two-issue cases. It is also different from the model of multiple bilateral negotiation between more than two players (e.g., [3]); it is a multilateral negotiation that always involves all players in a single negotiation. Thus, the negotiation model used by us is more involved and applies to multi-issue negotiation between more than two players in the real world. Given the model, we develop heuristics of negotiation strategies for three-agent two-issue cases where the agents have non-linear utility functions and incomplete information about his opponents' preferences, deadlines, etc. Compared to game-theoretic solutions, our heuristic methods are practical and tractable; the whole solution is very efficient such that (near) Pareto-optimal outcomes can be achieved. 
In our negotiation model, two issues are bundled and negotiated concurrently, so the agents can make trade-offs between issues when they have different preferences amongst the issues. This may generate win-win opportunities in which the agents reach an agreement that makes them mutually better off. However, this also makes the negotiation more complicated, because making appropriate proposals is nontrivial when an agent has multiple proposals which may give him the same maximum utility. To tackle with that, we decompose the negotiation strategies into two parts: a Pareto-optimalsearch method and concession strategies. The former takes the Pareto optimality into account to choose bids which can make other agents better off while keeping the bidder own desired utility. The latter determines the desired utilities that the agents want to get as the negotiation progresses.

In this work, we develop a Pareto-optimal-search method, which is called the orthogonal bidding strategy, for the three-agent multilateral negotiation. In [4-7], some similar methods work on two-agent cases where the desires of two agents, such as a buyer and a seller, are reverse to each other. That means, one agent wants to reach an agreement on large amounts of issues while the other one wants to reach an agreement on small amounts of issues. In the negotiation, they make compromises by moving their proposals towards each other. Each agent's proposal can be directly used to guide his opponent's counter-proposal towards an agreement. However, this becomes more difficult in our three-agent cases. One point is that all three agents here have the same directions of desires, getting large amounts of issues to achieve high utilities. Moving proposals close to each other directly is an inappropriate way to pursue an agreement. Another point is that compromises should take place between the desires of three sides. These two points make each agent difficult to determine his counter-proposal based on two proposals of his opponents directly. To solve the problem, first, we let each agent make bids only for his own parts of two issues [8]. Second, we introduce the reference point to model the bidding of the other two agents to guide the current bidder's decision. The reference points help the agents to choose the most satisfied bids for each other to pursue the Pareto optimality. The most satisfied bid made by an agent is the one benefiting his opponents most amongst multiple bids which give himself the same maximum utility. This is the cooperative part of a competitive game to generate win-win opportunities. Besides that, the reference points are also used to measure the distances from the current state to an agreement, which can be taken into account by the concession strategies, during the negotiation. Given a desired utility level and its corresponding indifference curve, the orthogonal bidding strategy lets an agent choose the bid which is closest (measured in the Euclidean distance) to the reference point introduced by the last bid of each of his opponents on the indifference curve. We show through computer experiments that by using the Pareto-optimal-search method for three-agent cases combined with well-designed concession strategies, the agents can reach agreements quickly under an incomplete information environment, and those outcomes are very close to Pareto-optimal solutions.

The rest of this paper is organized as follows. In Section 2, we give a brief summary of related work. In Section 3, we present the negotiation model including the problem model, the negotiation protocol and utility functions. In Section 4, we investigate the negotiation strategies, the orthogonal bidding strategy and various concession strate- 
gies, and discuss possible Pareto-optimal outcomes achieved by using our methods. In Section 5, we provide computer experiments to show the efficiency of our solutions. Conclusions and future work are given in Section 6.

\section{Related Work}

The complex negotiation has been extensively studied in last decades and most research work has been conducted in two fields, game theory and artificial intelligence (AI). In the game-theoretic way, the best known negotiation model is the alternating-offer bargaining game [9]. Its two-player single-issue negotiation model has been extended to the multi-player negotiation (e.g., $[2,10])$ or the multi-issue negotiation (e.g., $[1,11])$. The multi-player multi-issue negotiation has been studied in [8, 12]. A major limitation of the game-theoretic methods is that the equilibrium solutions are difficult to apply in practice, especially in the negotiation with incomplete information or non-linear utility functions or both. In this work, we adopt the multi-player multi-issue negotiation model proposed in [8], but propose heuristic computational methods of negotiation strategies of agents with incomplete information and non-linear utility functions.

Related to our work, in $[4,5]$, heuristic methods to find Pareto-optimal solutions of two-agent two-issue negotiation are introduced. The negotiation strategies are decomposed into concession strategies and Pareto-optimal-search methods. Given his opponent's offer, an agent uses concession strategies to determine his desired utility level first and then chooses a counter-offer on the indifference curve of the utility level in the orthogonal way, the counter-offer lying closest (measured in the Euclidean distance) to his opponent's offer. In [6,7], similar methods are used in both two-issue and threeissue cases. In these work of two-agent cases, an agent can directly regard his opponent's offer as the target to make compromises. It is easy to identify agreements and Pareto-optimal solutions based on two indifference curves. In our work, we propose an orthogonal method to solve the three-agent two-issue negotiation problem. When three agents partition two issues, any agent's offer cannot be used as a target to determine the counter-offer in the orthogonal way. We introduce the reference point to model every two agents' offers to support using the orthogonal method in three-agent cases. We also identify agreements and Pareto-optimal solutions based on three indifference curves, which is more complex than the situations in those work.

In [13], the agents apply similarity criteria to make trade-offs between issues. Given a desired utility level, an agent chooses a counter-offer on the indifference curve of the utility level that is most similar to his opponent's last offer. Their approach, however, is applied to linear additive utility functions and requires the agents have some knowledge about how their opponent weights the issues in the negotiation. In [14], the authors consider fuzzy constraints on the combination of multiple issues instead of the way that setting one fuzzy constraint on each single issue used in [13]. In [15], the authors propose a method based on kernel density estimation to learn the weights assigned by the opponent, but it still requires the agents have some prior information about the real weights the opponent assigns on the issues. Compared to their work, we consider the negotiation over continuous issues and choose the Euclidean distance instead the similarity criterion. It does not require any prior information of agents and is not restricted 
to linearly additive utility functions. In [16], the trade-off algorithm based on similarity criterion is extended to the negotiation between more than two agents. The algorithm lets each agent choose the counter-offer that is the most similar to the offers of all the other agents on his indifference curve. Because no single notion is introduced to model all the other offers, the algorithm lets the agent select the counter-offer as the one with minimum square (similarity) difference respecting all the other offers. In our work, we use one reference point to model two agents' bids and let the other agent still choose the closest offer based on the Euclidean distance. No other (new) measurement is required. Instead of the negotiation mechanism, an auction-based method is proposed in [17] for multi-issue negotiation with non-linear utility functions.

\section{The Negotiation Model}

Suppose three rational agents, $N=\{1,2,3\}$, partition two issues (resources), $M=$ $\{1,2\}$, through negotiation. The range of each issue is normalized to a continuous range $[0,1]$. Each agent requires a combination of a part of every issue. Hence, we let the two issues be bundled and negotiated concurrently, so the agents can make trade-offs between issues to maximize their utilities within an agreement. The negotiation takes place round by round $n \in \mathbb{N}$ until an agreement is reached or some agent quits. In each round, three agents bid partitions sequentially in some order. The bidding order can either be pre-specified before the negotiation starts or be generated randomly before every round. In this model, only a unanimous agreement can be accepted and implemented.

In the two-agent negotiation, whether one agent proposes partitions for two agents or only for himself has the same effect. However, if an agent needs to propose partitions for all in the negotiation between more than two agents, he has to decide how his opponents divide the issues left by him. Considering that every agent actually only focuses on his own parts and does not know the preferences of his opponents, we let agents bid in the way that every agent only proposes his own desired parts of issues [8]. We let $\mathbf{x}_{i} \in(0,1)^{2}$ denote a bid of agent $i \in N$ in a round and let $x_{i, j} \in \mathbf{x}_{i}$ denote the amount of issue $j \in M$. We define an agreement to be:

$$
\mathbf{x}=\left(\mathbf{x}_{1}, \mathbf{x}_{2}, \mathbf{x}_{3}\right) \text { subject to } \forall j \in M \sum_{i=1}^{3} x_{i, j} \leq 1
$$

where $\mathbf{x}$ is a profile of bids made by three agents in a round. If the three bids can form an agreement in the round, the negotiation stop successfully, otherwise the negotiation passes on to the next round. Each agent $i \in N$ has a negotiation deadline $T_{i}$. If no agreement is reached in any round $n \leq T$ where $T=\min \left\{T_{1}, T_{2}, T_{3}\right\}$, the agent will quit and the whole negotiation will stop unsuccessfully. Every agent gets zero utility at the outcome of disagreement.

In this work, we assume every agent has a strictly convex preference. We define the utility function $u_{i}:(0,1)^{2 \times 3} \times \mathbb{N} \rightarrow \mathbb{R}^{+}$, where $u_{i}(\mathbf{x}, n)$ represents the utility that agent $i$ would get in round $n$, if the agents all made bids as specified in $\mathbf{x}$. If $\mathbf{x}$ can form an agreement, the utility of $\mathbf{x}$ for agent $i$ only depends on his bid $\mathbf{x}_{i}$ and is calculated by a valuation function $v_{i}:(0,1)^{2} \rightarrow \mathbb{R}^{+}$. In this work, we assume the valuation function 
to be continuous and strictly monotonically increasing in each of the issues. Formally, the utility function is given by:

$$
u_{i}(\mathbf{x}, n)=\left\{\begin{aligned}
0 & \text { if } n>T \\
0 & \text { if } n=T \text { and not }(\mathbf{A}) \\
v_{i}\left(\mathbf{x}_{i}\right) & \text { if } n \leq T \text { and }(\mathbf{A})
\end{aligned}\right.
$$

where $\mathbf{x}_{i} \in \mathbf{x}$ and $T=\min \left\{T_{1}, T_{2}, T_{3}\right\}$.

In this model, every agent's preference and negotiation deadline are private information. Given the negotiation model above, we investigate the negotiation strategies of agents under the incomplete information setting.

\section{The Negotiation Strategies}

In this section, we present the negotiation strategies. As trade-offs exist between issues, it is possible that multiple bids give the same maximum utility to the bidder. Hence, when it is an agent's turn to bid, he can decide a desired utility level first and then choose a bid amongst those bids of the utility level. The negotiation strategy of an agent is then to specify $(i)$ how to determine the desired utility levels during the whole negotiation and (ii) how to bid given a desired utility level and possible bids of other agents.

Once an agent determines a desired utility level, all bids introducing the same maximum utility at the level are indifferent to the agent, but his bids may influence the bidding of his opponents, which results in various outcomes. To enhance the opportunity of reaching an agreement with his desired utility, the agent can choose a bid to benefit his opponents as much as possible at his current utility level. This is the cooperation part of the negotiation, in which all agents may be better off by making trade-offs between issues, and the negotiation may result in a Pareto-optimal outcome. An outcome is Pareto-optimal if there is no other outcome that would make at least on agent better off without making any other agent worse off. Given the multiple bids at an agent's current utility level, the one benefiting his opponents most will make them most satisfied. We call it the most satisfied bid. If no agreement can be reached on the current utility levels, even if all agents cooperatively make bids satisfying with each other as much as possible, one or more agents must concede their desires of utilities to pursue an agreement, which is better than zero utility of disagreement.

Hence, an agent's negotiation strategy can be decomposed into Pareto-optimalsearch methods and concession strategies [4]. The former takes the Pareto optimality into account to search the most satisfied bid (for all agents) on the current utility level; the latter is to determine how to make concessions by lowering the desired utility levels. Under an incomplete information setting, at any moment, every agent knows his own current desired utility level, his preference and the previous bids of his opponents. In the next section, first, we will present how to use the orthogonal bidding strategy as the Pareto-optimal-search method to make the most satisfied bid at the current utility levels in case of three agents. Second, we will introduce the concession strategies. 


\subsection{The Orthogonal Bidding Strategy}

Given the utility function of agent $i \in N$, a utility level can be represented by an indifference curve, which is a graph showing different combinations of issues, between which the agent is indifferent. Thus, it is equivalently to choose any bid (point) on an indifference curve as rendering the same level of utility for the agent. To enhance the opportunity to reach an agreement at his current desired utility level, the agent can choose one bid on the curve which satisfies his opponents most. That means, the rest of issues introduced by the bid gives his opponents highest possibility to get their desired utility levels. Reversely, if a bid of an agent is closest to the rest of issues left by his opponents, it is the one that his opponents satisfy most, give their current desires.

In this section, we will present how an agent determines the most satisfied bid on his curve, given the last bid of each of his opponents, and how the agents search the possible Pareto-optimal solutions, given their current desired utility levels.

The Strategy. When it is agent $i$ 's turn to bid, to choose a bid that satisfies his opponents most, he needs to model his opponents' desires first. For the two-agent negotiation studied in [4-7], each agent's offer can be used directly to guide his opponent's counter-offer towards an agreement. However, for the three-agent negotiation model in this work, moving bids close to each other cannot form an agreement. Moreover, when an agent considers compromises, two bids of his opponents should be taken into account together.

To solve the problem above, we introduce the reference point to represent the desires of any two agents for the other agent during the negotiation. Given two agents' bids, the rest of the issues composes a reference point which implies the joint desire of the two agents for the other agent, and making bids close to the reference point can make them satisfied. Formally, the reference point $r_{i}$ is given by

$$
r_{i}=\left(r_{i, 1}, r_{i, 2}\right)=\left(1-\sum_{k \in N-\{i\}} x_{k, 1}, 1-\sum_{k \in N-\{i\}} x_{k, 2}\right)
$$

where $\mathbf{x}_{k}=\left(x_{k, 1}, x_{k, 2}\right)$ is always the last bid of agent $k \in N-\{i\}$, which can happen in the current round or the previous round determined by the bidding order.

We define the orthogonal bidding strategy to be the way that when it is agent $i$ 's turn to bid, he always chooses the bid on his current indifferent curve which is closest (measured in the Euclidean distance) to the reference point $r_{i}$. In this work, the reference points are given by Equation (2). We mention that there may be some other ways to choose the reference points, and the way to determine the reference point is independent of the orthogonal bidding strategy. Figure 1 illustrates how each agent chooses a bid most satisfied his opponents' desires, which are modeled by a reference point based on their latest bids, where no concession happens during the negotiation round (so the curves are fixed). The indifference curves of the example are generated based on CobbDouglas functions. Given the bids of agent 1 and 3 in round $n$, agents 2, 3 and 1 bid sequentially in round $n+1$.

(1) First agent 2 calculates the reference point based on the bids of agent 1 and 3 in round $n$. 


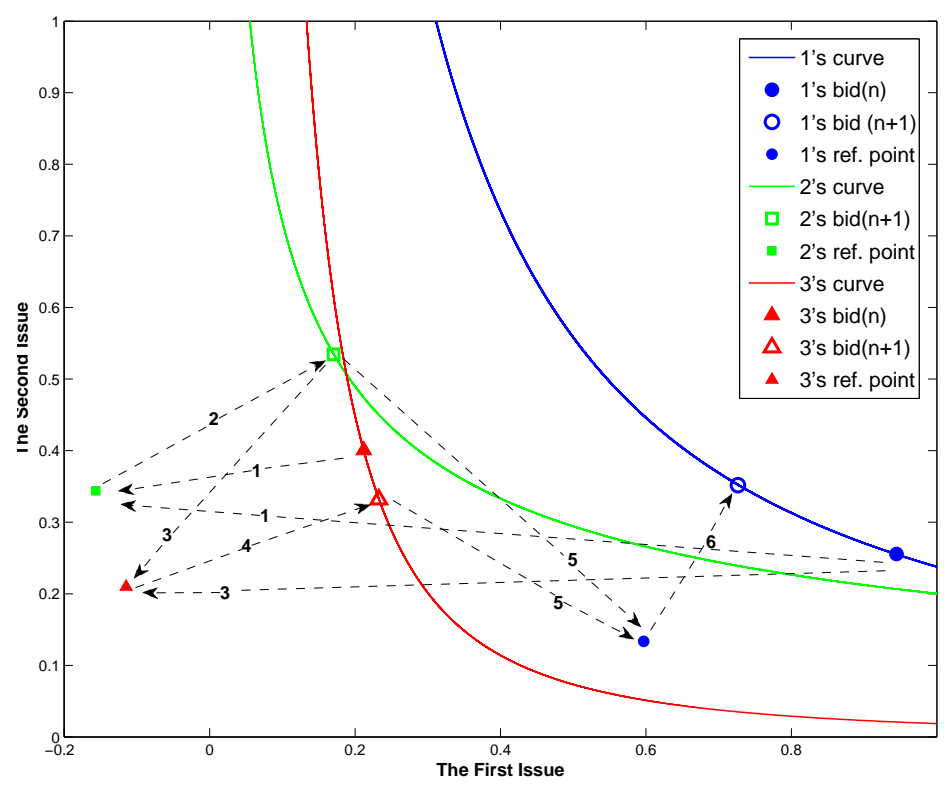

Fig. 1. Example of the orthogonal bidding strategy

(2) Agent 2 chooses the bid which is closest to the reference point determined in step (1) on his curve.

(3) Agent 3 calculates the reference point based on agent 1's bid in round $n$ and agent 2 's bid chosen in step (2).

(4) Agent 3 chooses the bid which is closest to the reference point determined in step (3) on his curve.

(5) Agent 1 calculates the reference point based on agent 2's bid chosen in step (2) and agent 3's bid chosen in step (4).

(6) Agent 1 chooses the bid which is closest to the reference point determined in step (5) on his curve.

Although it is possible that some agent uses the orthogonal bidding strategy but some agent uses another negotiation strategy, in the rest of this work, we analyze the solution that all three agents use the orthogonal bidding strategy to search the Pareto-optimal solution. The analysis also holds if they do not know the strategies of each other, but they do use the orthogonal bidding strategy.

The Agreement. To better to describe the process of reaching an agreement, we let $R$ be the point representing the remaining issues. given three bids, and let $\Delta$ denote the distance between the remaining issues $R$ to the origin $\mathbf{0}=(0,0)$. Formally, the 
remaining issues $R$ and the distance $\Delta$ can be calculated by

$$
\begin{aligned}
& R=\left(R_{1}, R_{2}\right)=\left(1-\sum_{i=1}^{3} x_{i, 1}, 1-\sum_{i=1}^{3} x_{i, 2}\right), \\
& \Delta=\|R-\mathbf{0}\|=\|R\|
\end{aligned}
$$

The remaining issues $R$ indicates the bidding result of a round, given three bids; the reference point $r_{i}$ indicates the bidding state of agent $i$ 's opponents, given two bids. Note that before reaching an agreement, the remaining issues here may have negative values, $R_{1}<0$ or $R_{2}<0$ or both, which implies the total requirements of issues still need to be reduced. In case the remainder is negative, we will use the measure $\Delta$ to indicate how far the current negotiation state is from an agreement. Next, we use an example to show how three agents keep using the orthogonal bidding strategy on fixed utility levels to reach an agreement. We call the process the orthogonal search. In this example, three agents' initial bids are generated randomly and the indifference curves are generated based on Cobb-Douglas functions. Figure 2 illustrates the orthogonal

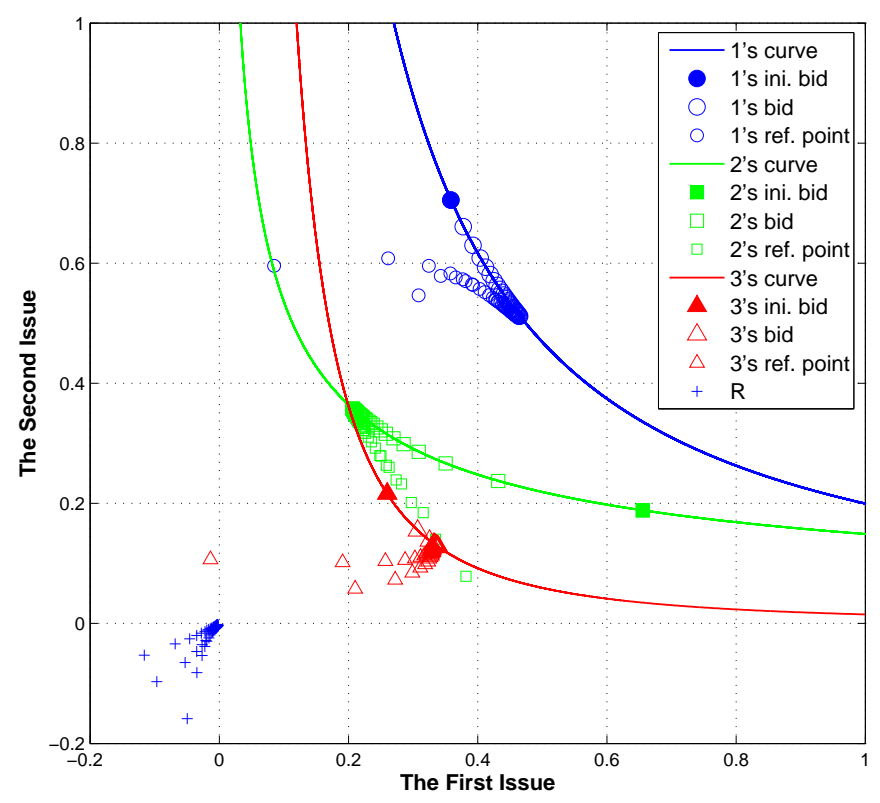

Fig. 2. Example of the orthogonal search

search in which three agents keep bidding on their utility levels without concession. The bids and reference points move closer round by round. Finally, each agent's bids 
and reference points are converged into one point on his indifference curve. That means, each agent's final bid completely satisfies the desires of his opponents and an agreement is reached. From another perspective, the distance $\Delta$ keeps reducing and the remaining issues $R$ moves to the origin $\mathbf{0}$ and finally converges into it. That also indicates reaching an agreement. We mention it is possible that the bids of an agreement do not exactly coincide with their reference points after finite rounds, and the convergence may be generated based on the finite precision of computers. Based on the example, we give the following lemma.

Lemma 1. A profile of bids $\mathbf{x}=\left(\mathbf{x}_{1}, \mathbf{x}_{2}, \mathbf{x}_{3}\right)$ is an agreement $\Longleftrightarrow$ each reference point $r_{i}$ Pareto dominates bid $\mathbf{x}_{i}$, i.e., $r_{i, j} \geq x_{i, j}$, where $i \in N$ and $j \in M$.

Proof. Because $\mathbf{x}$ is an agreement, given the condition of agreements (A), $x_{i, j} \leq 1-$ $\sum_{k \in N-\{i\}} x_{k, j}$ where $j \in M$. Given the definition of reference points in Equation (1), $r_{i}$ Pareto dominates $\mathbf{x}_{i}$, i.e., $r_{i, j} \geq x_{i, j}$.

If $r_{i, j} \geq x_{i, j}$ where $i \in N$ and $j \in M$, given the definition of reference points in Equation (1), we have $x_{i, j}+\sum_{k \in N-\{i\}} x_{k, j} \leq 1$. Hence, the bid profile $\mathbf{x}$ is an agreement.

The Pareto Optimality. In the example of Figure 2, the remaining issues $R$ converges into the origin. That means, all issues are exactly partitioned without shortages and remains. However, this does not necessarily indicate a Pareto-optimal solution in multiissue negotiation as the agents may still have chances to get Pareto improvements by making trade-offs between issues. A Pareto improvement is a change from one solution to another solution that can make at least one agent better off without sacrificing the utilities of the other agents. The following example shows a negotiation solution, in which the issues are partitioned completely but the opportunities of Pareto improvements clearly exist.

Figure 3 illustrates an example where three agents reach an agreement by using the orthogonal search on relatively low utility levels. The initial reference points in Figure 3 are derived from the final reference points of the orthogonal search on higher utility levels. Each agent determines his first bid based on the initial reference points in the orthogonal way separately and then do the orthogonal search on the fixed curves. We observe that each agent's bids and reference points converge into one point (in the direction indicated by the arrow) on the curve and an agreement is formed by the convergence points. (The convergence point of agent 2 just happens close to his first bid.) However, different from the example in Figure 2, the reference points appear in the upper-right area of the curves. Because those indifference curves are strictly convex, any point (bid) in the upper-right area of a curve can give the agent a higher utility than the utility level of the curve. Suppose agent $i$ chooses a reference point $r_{i}$ in the upperright area of his curve instead of the convergence point as his bid and the other two agents choose the bids which generate $r_{i}$ on their curves. Each of the new bids equals to the reference point introduced by the other two bids. The three new bids can form an agreement (Lemma 1); agent $i$ 's utility is increased and the other two agents' utilities are unchanged. That is a Pareto improvement, so the solution of the three convergence points is not Pareto-optimal. From another perspective, except for the final state, the 


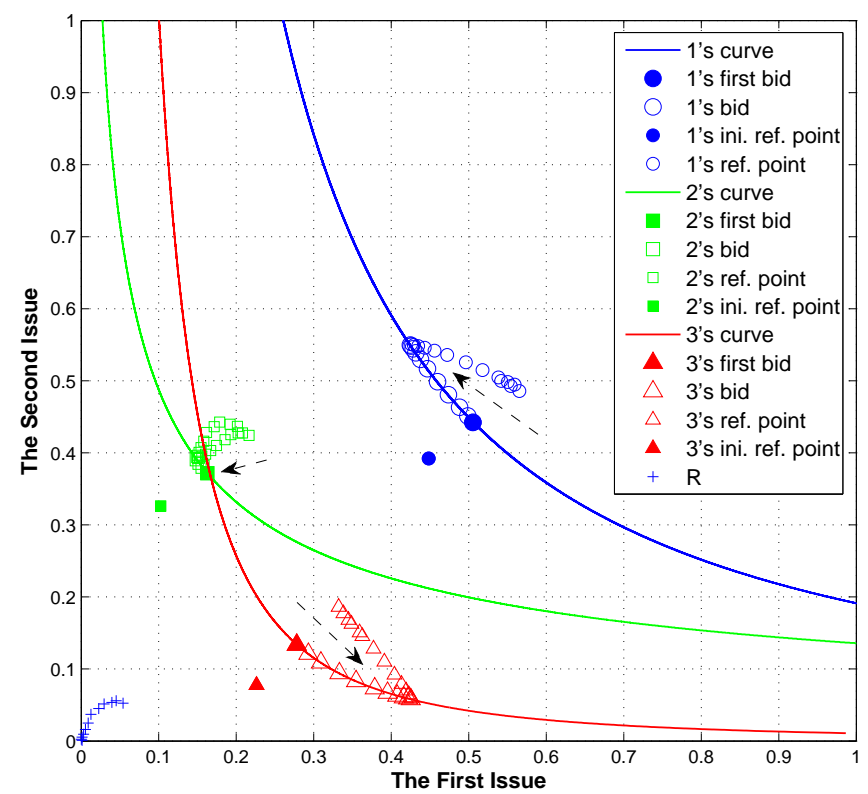

Fig. 3. Example of the orthogonal search on low utility levels

remaining issues are always more than zero in the orthogonal search. That means, the agents have opportunities to reach an agreement with less issue and use extra issues to make Pareto improvement. Next, we give the geometrical interpretation of Paretooptimal solutions achieved by using our method and the mathematical proof.

We let $c_{i}$ denote the current desired utility level of agent $i \in N$ and $C_{i}$ denote the set of all points on the indifference curve of utility $c_{i}$, i.e., $C_{i}=\left\{\mathbf{x}_{i} \mid v_{i}\left(\mathbf{x}_{i}\right)=c_{i}\right\}$. That means, any point in $C_{i}$ has the value $c_{i}$. If the point is a bid of an agreement, the bid will give agent $i$ utility $c_{i}$. Every combination of two points (bids) on the other two agents' curves introduces a reference point $r_{i}$ to agent $i$. Given any reference point $r_{i}$ where $r_{i, j}>0(j \in M)$, if agent $i$ chooses it as his bid, each of his opponents can get a utility at least as good as his current desired utility and agent $i$ can get a utility larger than zero. Given the area composed of all such reference points, we call it the reference area of agent $i$ where every bid (point) in the area can satisfy his opponents. We let $X_{i}$ denote the set of all such bids of agent $i$, i.e., $X_{i}=\left\{\mathbf{x}_{i} \mid \forall k \neq i \exists \mathbf{x}_{k}\right.$ such that $v_{k}\left(\mathbf{x}_{k}\right) \geq c_{k}$ and $\left.(\mathbf{A})\right\}$. (Recall that $\mathbf{x}_{i} \in(0,1)^{2}$.) Because indifference curves are strictly convex, for each other agent $k \in N-\{i\}$, the set of all agreements where agent $k$ gets at least utility $c_{k}$ is a convex set (i.e., the area above the indifference curve), and $X_{i}$ is the intersection of these sets, one for each other agent $k$, of all agreements where agent $k$ gets at least utility $c_{k}$, and is thus also a convex set. 
Lemma 2. $\left|X_{i} \cap C_{i}\right|>1$ where $i \in N \Longleftrightarrow$ there is an agreement $\mathbf{x}$ such that $u_{i}(\mathbf{x}, n)>c_{i}(n \leq T)$.

Proof. Suppose $\left|X_{i} \cap C_{i}\right|>1$. Let $\mathbf{y}_{i} \neq \mathbf{z}_{i}$ be two points in $X_{i} \cap C_{i}$. Let $\mathbf{x}_{i}$ be a convex combination of $\mathbf{y}_{i}$ and $\mathbf{z}_{i}$, i.e., for a $t \in(0,1)$ let $\mathbf{x}_{i}=t \cdot \mathbf{y}_{i}+(1-t) \cdot \mathbf{z}_{i}$. Because the indifference curve is strictly convex, $\mathbf{x}_{i}$ lies above the curve, thus $v_{i}\left(\mathbf{x}_{i}\right)>c_{i}$. Furthermore, by convexity of $X_{i}$, it follows that $\mathbf{x}_{i} \in X_{i}$, so there exists an agreement $\mathbf{x}$ where $\mathbf{x}_{i} \in \mathbf{x}$ and $u_{i}(\mathbf{x}, n)=v_{i}\left(\mathbf{x}_{i}\right)>c_{i}$ and $n \leq T$.

To prove the converse, suppose now that there is an agreement $\mathbf{x}$ where $\mathbf{x}_{i} \in \mathbf{x}$ and $u_{i}(\mathbf{x}, n)=v_{i}\left(\mathbf{x}_{i}\right)>c_{i}$. Let $\mathbf{y}_{i}=\left(y_{i, 1}, y_{i, 2}\right)$ be the point with utility $c_{i}$ such that $y_{i, 1}=x_{i, 1}$, and let $\mathbf{z}_{i}=\left(z_{i, 1}, z_{i, 2}\right)$ be the point with utility $c_{i}$ such that $z_{i, 2}=x_{i, 2}$. These points both exist, because of continuity of the utility function. Also we know that $\mathbf{y}_{i}$ and $\mathbf{z}_{i}$ are in $X_{i}$ because they only use less of one of the issues, and all utility functions are strictly monotonically increasing in all issues. Thus $X_{i} \cap C_{i}$ contains at least these two points.
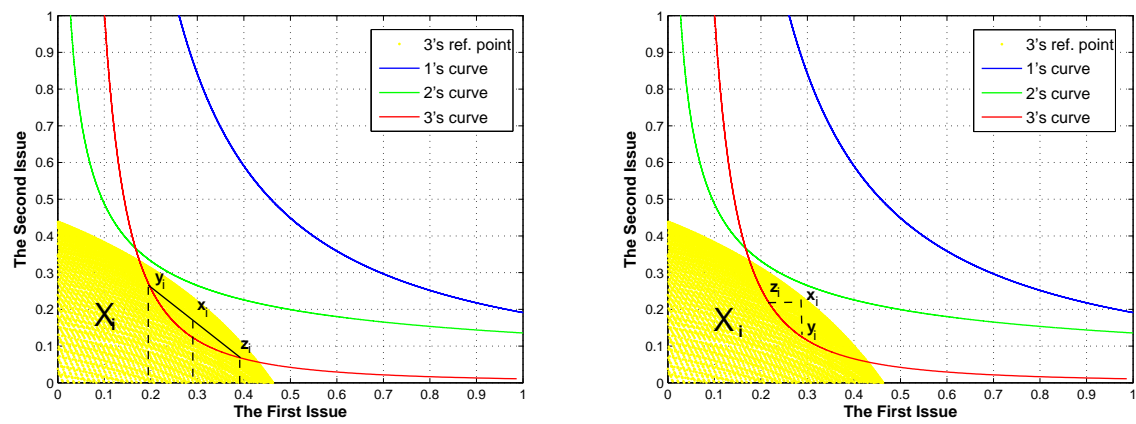

Fig. 4. Example of the reference area

Figure 4 gives the graphical representation of Lemma 2 where agent $i$ represents the third agent. The left one illustrates the existence of an agreement $\mathbf{x}$ where $\mathbf{x}_{i} \in \mathbf{x}$ is above the curve and $\mathbf{x}_{i}$ is a convex combination of $\mathbf{y}_{i}$ and $\mathbf{z}_{i}$, when $\left|X_{i} \cap C_{i}\right|>1$. The right one illustrates when an agreement $\mathbf{x}$ exists and $u_{i}(\mathbf{x}, n)=v_{i}\left(\mathbf{x}_{i}\right)>c_{i}$, there are at least two points $\mathbf{y}_{i}, \mathbf{z}_{i} \in X_{i}$ on the curve. Hence, $\left|X_{i} \cap C_{i}\right|>1$.

Obviously, when an agreement $\mathbf{x}$ in the above example exists, agent $i$ (red one) can make Pareto improvements by bidding $\mathbf{x}_{i}$ instead of any point on his current indifference curve. Reversely, if $X_{i} \cap C_{i}=\emptyset$, no agreement can be reached on the current utility levels, which is not a Pareto-optimal outcome of this negotiation. Hence, if the reference area and the indifference curve of every agent $i \in N$ has a unique intersection, the profile of three intersections is a Pareto-optimal solution. We give the following theorem with a condition that the indifference curves are strictly convex.

Theorem 1. $\left|X_{i} \cap C_{i}\right|=1$ where $i \in N \Longleftrightarrow$ there is a Pareto-optimal solution $\mathbf{x}=\left(\mathbf{x}_{1}, \mathbf{x}_{2}, \mathbf{x}_{3}\right)$ where $u_{i}(\mathbf{x}, n)=c_{i}(n \leq T)$. 
Proof. We give proof by contradiction. Suppose there is a Pareto-optimal solution $\mathbf{x}$ where $u_{i}(\mathbf{x}, n)=c_{i}$. Suppose $\left|X_{i} \cap C_{i}\right| \neq 1$. If $\left|X_{i} \cap C_{i}\right|=0$, no agreement can be reached at every agent $i$ 's desired utility level $c_{i}$, so no Pareto-optimal solution $\mathbf{x}$ exists. There is a contradiction. If $\left|X_{i} \cap C_{i}\right|>1$, Pareto improvements exist, i.e., $\exists \mathbf{x}^{\prime}$ such that $u_{i}\left(\mathbf{x}^{\prime}, n\right)>c_{i}$ (Lemma 2), so the solution $\mathbf{x}$ in which $u_{i}(\mathbf{x}, n)=c_{i}$ is not a Pareto-optimal solution. There is a contradiction. Hence, if there is a Pareto-optimal solution $\mathbf{x}$ where $u_{i}(\mathbf{x}, n)=c_{i}$, then $\left|X_{i} \cap C_{i}\right|=1$ and $X_{i} \cap C_{i}=\left\{\mathbf{x}_{i}\right\}$.

Suppose $\left|X_{i} \cap C_{i}\right|=1$. Suppose no Pareto-optimal solution exists. If no agreement exists, then $\left|X_{i} \cap C_{i}\right|=0$. There is a contradiction. If there is an agreement $\mathbf{x}$ but it is not Pareto-optimal, then Pareto improvements exists. If $\exists \mathbf{x}^{\prime}$ such that $u_{i}\left(\mathbf{x}^{\prime}, n\right)>c_{i}$, then $\left|X_{i} \cap C_{i}\right|>1$ (Lemma 2). There is a contradiction. Hence, if $\left|X_{i} \cap C_{i}\right|=1$, there is a Pareto-optimal solution $\mathbf{x}$ where $X_{i} \cap C_{i}=\left\{\mathbf{x}_{i}\right\}$ and $u_{i}(\mathbf{x}, n)=c_{i}$.

\subsection{Concession Strategies}

In the previous section, we presented how the agents bid at desired utility levels. At the beginning of the negotiation, each agent has a relatively high desire of utility, and he can choose to concede at any moment as the negotiation progresses. In this section, we address the concession strategies which determine how to concede, given the previous bids. In this work, we develop four concession strategies:

1. Amount of utility. Agent $i \in N$ concedes a fixed amount utility au.

2. Fraction of utility. Agent $i \in N$ concedes a fixed fraction $f u<1$ of the current desired utility.

3. Fraction of difference. Agent $i$ concedes a fixed fraction $f d<1$ of the difference between his current desired utility and the utility that he would get by bidding the last reference point $r_{i}$. When $r_{i, j}<0(j \in M)$, the strategy adopts the difference between his current desired utility and zero utility, which means using the origin to replace the reference point $r_{i}$.

4. Fraction of remains. Agent $i$ concedes a fix fraction $f r<1$ of the remaining issues $R$. Before reaching an agreement, one or two issues' remains $R_{j}$ have negative values. The conceding is to add a fraction of the remain(s) that has the negative value(s) to the last bid $\mathbf{x}_{i}$.

For the first strategy, the concession per time is fixed. For all the other three strategies, the concession per time turns to small as the negotiation proceeds, which is helpful for agents to reach (near) Pareto-optimal solutions.

Combining the concession strategies and the orthogonal bidding strategy, in next section, we evaluate the average performance of different combinations of negotiation strategies. We will measure the Pareto improvement of each agent, the change from his utility and bid in the agreement to his utility and bid in a Pareto-optimal solution.

\section{Experimental Analysis}

In this section, we design experiments to measure the efficiency and optimality of the proposed negotiation solutions, and compare the performance of different concession 
strategies. In the setting we study, there is a negotiation deadline for each of the agents, and therefore some incentive to make concessions. In our current experiments we let the agents make concessions after each round until an agreement is reached, and leave a more detailed analysis of strategic behavior for future work.

\subsection{Settings}

We simulate the negotiation with two types of functions that are used a lot in economics: (i) the Cobb-Douglas function and $(i i)$ the constant elasticity of substitution (CES) function. Given the definition of utility function (1), we specify the valuation function $v_{i}(i \in N)$ by:

(i) Cobb-Douglas function $v_{i}\left(\mathbf{x}_{i}\right)=\gamma_{i} \cdot x_{i, 1}^{\alpha_{i}} \cdot x_{i, 2}^{\beta_{i}}$,

(ii) CES function $v_{i}\left(\mathbf{x}_{i}\right)=\gamma_{i} \cdot\left(\alpha_{i} \cdot x_{i, 1}^{\beta_{i}}+\left(1-\alpha_{i}\right) \cdot x_{i, 2}^{\beta_{i}}\right)^{1 / \beta_{i}}$.

We generate the parameters of the valuation functions randomly and choose five typical cases listed in Table 1. Therefore, we have 5 instances of utility functions in the fol-

Table 1. Parameters of utility functions

\begin{tabular}{|c|ccc|ccc|ccc|}
\hline Type & $\alpha_{1}$ & $\alpha_{2}$ & $\alpha_{3}$ & $\beta_{1}$ & $\beta_{2}$ & $\beta_{3}$ & $\gamma_{1}$ & $\gamma_{2}$ & $\gamma_{3}$ \\
\hline \hline Cobb-Douglas 1 & 0.64 & 0.65 & 0.83 & 0.52 & 0.83 & 0.42 & 5.97 & 6.21 & 8.14 \\
\hline Cobb-Douglas 2 & 0.24 & 0.25 & 0.25 & 0.92 & 0.78 & 0.78 & 6.4 & 7.9 & 8.5 \\
\hline Cobb-Douglas 3 & 0.267 & 0.35 & 0.23 & 0.91 & 0.82 & 0.88 & 5.31 & 5.34 & 8.31 \\
\hline CES 1 & 0.91 & 0.855 & 0.83 & 0.123 & 0.25 & 0.33 & 8.23 & 7.12 & 9.28 \\
\hline CES 2 & 0.717 & 0.65 & 0.623 & 0.443 & 0.492 & 0.464 & 7.8 & 8.72 & 9.348 \\
\hline
\end{tabular}

lowing experiments. Given four concession strategies, for each of them, we choose two factors. One is a (relatively) big step factor, which can make the agents reach agreements quickly and get near Pareto-optimal outcomes. The other one is a small step factor, which let agents get more efficient outcomes. Therefore, we totally have 8 instances of concession strategies.

Given an agreement $\mathbf{x}$, we measure the efficiency of this outcome by Pareto improvements. For each agent $i \in N$, we let $P_{i}$ denote the Euclidean distance between his final bid $\mathbf{x}_{i} \in \mathbf{x}$ and his bid in the Pareto-optimal solution, in which agent $i$ 's utility is maximized and the utility for agent $k \in N-i$ is kept the same as $u_{k}(\mathbf{x}, n)$ where $n$ is the finish round of negotiation. In addition, we let $Q_{i}$ denote the difference between agent $i$ 's utility $u_{i}(\mathbf{x}, n)$ and his utility achieved in the Pareto-optimal solution.

For each combination of one utility function instance and one concession strategy instance, we run the negotiation 100 times, each of which has random initial bids. Given all results using instances of Cobb-Douglas utility functions, we calculate average $P_{i}$ and $Q_{i}$ for each concession strategy instance. Finally, we get 8 pairs of $P_{i}$ and $Q_{i}$ of each agent $i$ for 8 concession strategy instances combined with Cobb-Douglas utility functions, and get another 8 pairs of $P_{i}$ and $Q_{i}$ of each agent $i$ for 8 concession strategy 
instances combined with CES utility functions. To better compare and analyze the performance of our solutions, we enlarge the range of issues 100 times in the calculation of utilities. All results are presented in the next section.

\subsection{Experimental Results}

Table 2 shows the average Euclidean distance $P_{i}$ and utility difference $Q_{i}$ from the Pareto-optimal solution of each agent $i$ for various concession strategy instances combined with Cobb-Douglas utility functions. Table 3 shows the average Euclidean distance $P_{i}$ and utility difference $Q_{i}$ from the Pareto-optimal solution of each agent $i$ for various concession strategy instances combined with CES utility functions. For example, the element $(1,1)(=0.0541 / 25.6880)$ in Table 2 is the average Euclidean distance and utility difference of agent 1 between his bids in the agreements and the Pareto-optimal solutions respectively, which are generated in the experiments of concession strategy "amount of utility" with the factor $a u=30$ and Cobb-Douglas utility functions. The column of round in each table indicates how many rounds the agents reach the agreements.

Table 2. Average Euclidean distance and utility difference from Pareto-optimal solutions of each agent for various concession strategy instances combined with Cobb-Douglas utility functions

\begin{tabular}{|c|c|c|c|c|}
\hline Concession strategy & $P_{1} / Q_{1}$ & $P_{2} / Q_{2}$ & $P_{3} / Q_{3}$ & Round \\
\hline \hline Amount of utility $(a u=30)$ & $0.0541 / 25.6880$ & $0.0314 / 34.3712$ & $0.0383 / 32.6222$ & 11 \\
\hline Amount of utility $(a u=10)$ & $0.0103 / 8.3359$ & $0.0127 / 11.4980$ & $0.0117 / 10.5680$ & 21 \\
\hline Frac. of utility $(f u=0.05)$ & $0.0312 / 25.2408$ & $0.0301 / 30.2793$ & $0.0279 / 28.1602$ & 12 \\
\hline Frac. of utility $(f u=0.01)$ & $0.0121 / 8.1018$ & $0.0096 / 11.0645$ & $0.0132 / 9.0251$ & 58 \\
\hline Frac. of difference $(f d=0.1)$ & $0.0190 / 1.7297$ & $0.0164 / 2.6683$ & $0.0139 / 1.9356$ & 17 \\
\hline Frac. of difference $(f d=0.05)$ & $0.0070 / 1.2109$ & $0.0072 / 1.6012$ & $0.0090 / 1.2351$ & 31 \\
\hline Frac. of remains $(f r=0.15)$ & $0.0830 / 14.8853$ & $0.0724 / 15.4760$ & $0.0451 / 13.9908$ & 9 \\
\hline Frac. of remains $(f r=0.1)$ & $0.0438 / 3.4280$ & $0.0372 / 5.1806$ & $0.0168 / 4.0223$ & 15 \\
\hline
\end{tabular}

Table 3. Average Euclidean distance and utility distance from Pareto-optimal solutions of each agent for various concession strategy instance combined with CES utility functions

\begin{tabular}{|c|c|c|c|c|}
\hline Concession strategy & $P_{1} / Q_{1}$ & $P_{2} / Q_{2}$ & $P_{3} / Q_{3}$ & Round \\
\hline \hline Amount of utility $(a u=30)$ & $0.0725 / 44.9505$ & $0.0711 / 41.0294$ & $0.0795 / 50.9900$ & 15 \\
\hline Amount of utility $(a u=10)$ & $0.0372 / 14.2733$ & $0.0261 / 14.8818$ & $0.0283 / 16.9309$ & 43 \\
\hline Frac. of utility $(f u=0.05)$ & $0.0413 / 23.2897$ & $0.0401 / 23.0080$ & $0.0422 / 27.0832$ & 19 \\
\hline Frac. of utility $(f u=0.01)$ & $0.0330 / 7.3399$ & $0.0136 / 7.2989$ & $0.0220 / 8.5175$ & 93 \\
\hline Frac. of difference $(f d=0.1)$ & $0.0209 / 2.0875$ & $0.0139 / 1.9603$ & $0.0402 / 2.3787$ & 19 \\
\hline Frac. of difference $(f d=0.05)$ & $0.0037 / 1.3575$ & $0.0231 / 1.1985$ & $0.0168 / 1.5789$ & 35 \\
\hline Frac. of remains $(f r=0.15)$ & $0.0152 / 4.1480$ & $0.0561 / 4.0198$ & $0.0623 / 4.6728$ & 11 \\
\hline Frac. of remains $(f r=0.1)$ & $0.0308 / 2.1478$ & $0.0317 / 1.9390$ & $0.0369 / 2.3900$ & 16 \\
\hline
\end{tabular}


From the results, we find both the Euclidean distance and the utility difference from the agreements to the Pareto-optimal solutions of each concession strategy instance is very small, which indicates our solution can provide near Pareto-optimal solutions for those types of utility functions. (Recall that the range of issues is enlarged 100 times in the calculation of utilities.) For each concession strategy, when a big step factor is used, the agreements can be reached very fast; when a small step factor is used, the agreements are very close to the Pareto-optimal solutions for every agents. Although the performance of the former is a little lower than that of the latter, we find it is also very good. Meanwhile, the speed of reaching agreements of the latter is a little slower than that of the former, but it is completely acceptable in the automated negotiation between software agents. Amongst those concession strategies, we find the performance of concession strategy "fraction of difference" is best in our experiments. The reason is it takes the reference points into account, which is the key factor of the orthogonal bidding strategy. To conclude, the solution of the orthogonal bidding strategy combined with well-designed concession strategies is very efficient such that near Pareto-optimal outcomes can be achieved quickly.

\section{Conclusions and Future Work}

In this paper, we present an automated three-agent two-issue negotiation solution to solve a resource allocation problem. We adopt a multilateral negotiation model, in which three agents bid combinations of parts of two continuously valued issues sequentially. The model applies to multi-issue negotiation between more than two players in the real world. We develop tractable heuristics of negotiation strategies used in a complex setting, in which the agents have non-linear utility functions and incomplete information about their opponents' preferences and negotiation deadlines. The negotiation strategies are decomposed into two parts: a Pareto-optimal-search method and concession strategies. The agents determine their desired utility levels by using concession strategies first and then use the orthogonal bidding strategy as the Pareto-optimalsearch method to find the bids which satisfy all agents most on their current utility levels. The method makes bids approaching the Pareto-optimal solution as the negotiation proceeds. Important technique contributions lie in the development of the orthogonal bidding strategy for three-agent cases, which can be easily extended to multi-agent cases, and the identification of agreements and Pareto-optimal outcomes achieved by the methods based on mathematical proof. We show through computer experiments that the solution composed of the orthogonal bidding strategy and well-designed concession strategies is very efficient such that (near) Pareto-optimal outcomes can be achieved in short time.

An interesting extension of our work will be to develop the orthogonal bidding strategy for three-agent three-issue cases. The orthogonal method will work on indifference surfaces. Searching the most satisfied bids and identifying agreements and Paretooptimal outcomes will become more complex. Further, we may design some heuristic combined with the orthogonal method to solve the negotiation with many issues. Another interesting extension is to develop more precise concession strategies, such as 
time independent strategies and correlated strategies. Moreover, we may study the negotiation results when utility functions are non-strict monotonicity.

\section{References}

1. Fatima, S., Wooldridge, M., Jennings, N.: Multi-Issue Negotiation with Deadlines. Journal of Artificial Intelligence Research 27 (2006) 381-417

2. Binmore, K.: Bargaining and Coalitions. Game-Theoretic Models of Bargaining (1985) 269-304

3. Gerding, E., Somefun, D., La Poutre, J.: Multi-attribute Bilateral Bargaining in a One-toMany Setting. LECTURE NOTES IN COMPUTER SCIENCE 3435 (2005) 129

4. Somefun, D., Gerding, E., Bohte, S., La Poutre, J.: Automated negotiation and bundling of information goods. Lecture notes in computer science (2004) 1-17

5. Somefun, D., Gerding, E., La Poutre, J.: Efficient methods for automated multi-issue negotiation: Negotiating over a two-part tariff. International Journal of Intelligent Systems 21(1) (2006)

6. Lai, G., Sycara, K., Li, C.: A decentralized model for multi-attribute negotiations with incomplete information and general utility functions. In: Proceedings of second international workshop on rational, robust, and secure negotiations in multi-agent systems, Springer (2006)

7. Lai, G., Sycara, K.: A generic framework for automated multi-attribute negotiation. Group Decision and Negotiation (2009)

8. Wu, M., de Weerdt, M., La Poutré, J., Yadati, C., Zhang, Y., Witteveen, C.: Multi-player Multi-issue Negotiation with Complete Information. In: Proceedings of second international workshop on agent-based complex automated negotiations, Springer (2009)

9. Rubinstein, A.: Perfect Equilibrium in a Bargaining Model. Econometrica 50(1) (1982) $97-110$

10. Shaked, A.: A Three-Person Unanimity Game. Talk given at the Los Angeles national meetings of the Institute of Management Sciences and the Operations Research Society of America (1986)

11. Fatima, S., Wooldridge, M., Jennings, N.: An agenda-based framework for multi-issue negotiation. Artificial Intelligence 152(1) (2004) 1-45

12. Rausser, G., Simon, L.: A non-cooperative model of collective decision making: A multilateral bargaining approach. Department of Agricultural and Resource Economics (University of California, Berkeley) (1992)

13. Faratin, P., Sierra, C., Jennings, N.: Using similarity criteria to make issue trade-offs in automated negotiations. Artificial Intelligence 142(2) (2002) 205-237

14. Luo, X., Jennings, N., Shadbolt, N., Leung, H., Lee, J.: A fuzzy constraint based model for bilateral, multi-issue negotiations in semi-competitive environments. Artificial Intelligence 148(1) (2003) 53-102

15. Coehoorn, R., Jennings, N.: Learning on opponent's preferences to make effective multiissue negotiation trade-offs. In: Proceedings of the 6th international conference on Electronic commerce, ACM New York, NY, USA (2004) 59-68

16. Ros, R., Sierra, C.: A negotiation meta strategy combining trade-off and concession moves. Autonomous Agents and Multi-Agent Systems 12(2) (2006) 163-181

17. Ito, T., Hattori, H., Klein, M.: Multi-issue negotiation protocol for agents: Exploring nonlinear utility spaces. In: Proceedings of the 20th International Joint Conference on Artificial Intelligence (IJCAI07). (2007) 1347-1352 